\title{
Barriers to Postpartum Glucose Intolerance Screening in an Italian Population
}

\author{
Paola Quaresima ${ }^{1,+}{ }^{+}$Federica Visconti ${ }^{1,+}$, Eusebio Chiefari ${ }^{2}$, Luigi Puccio ${ }^{3}$, Daniela P. Foti ${ }^{2}$, \\ Roberta Venturella ${ }^{1}$, Raffaella Vero ${ }^{3}$, Antonio Brunetti ${ }^{2}{ }^{-1}$ and Costantino Di Carlo ${ }^{1, *}$ \\ 1 Unit of Obstetrics and Gynecology, Department of Medical and Surgical Sciences, University “Magna \\ Græcia" of Catanzaro, Viale Europa, 88100 Catanzaro, Italy; dr.paolaquaresima@gmail.com (P.Q.); \\ fed.visconti@gmail.com (F.V.); rovefa@libero.it (R.V.) \\ 2 Department of Health Sciences, University "Magna Græcia" of Catanzaro, Viale Europa, 88100 Catanzaro, \\ Italy; echiefari@libero.it (E.C.); foti@unicz.it (D.P.F.) brunetti@unicz.it (A.B.) \\ 3 Complex Operative Structure Endocrinology-Diabetology, Hospital Pugliese-Ciaccio, 88100 Catanzaro, Italy; \\ puccio55@libero.it (L.P.); rafvero@libero.it (R.V.) \\ * Correspondence: cdicarlo@unicz.it \\ + These authors contributed equally to this work.
}

Received: 13 November 2018; Accepted: 11 December 2018; Published: 14 December 2018

\begin{abstract}
Background: Gestational diabetes mellitus (GDM) is a strong risk factor for type 2 diabetes mellitus (T2D) and the postpartum period is crucial for early treatment in at-risk women. However, despite recommendations, only a fraction of women undergo a postpartum screening for glucose intolerance (ppOGTT). The present study aims to verify the reason(s) for poor adherence in our population. Research design and methods: This retrospective study includes 451 women in which GDM was diagnosed between 2015-2016. During 2017, we verified by phone interview how many women underwent pPOGTT at 6-12 weeks postpartum, as recommended by the Italian guidelines. The non-compliant women were asked about the reason(s) for failing to screen. The non-parametric Mann-Whitney test and the 2-tailed Fisher exact test were used to compare continuous and categorical features, respectively, among women performing or non-performing ppOGTT. Results: Out of 451 women with GDM diagnosis, we recorded information from 327. Only $97(29.7 \%)$ performed ppOGTT. The remaining 230 women (70.3\%) provided the following explanation for non-compliance: (1) newborn care $(30.4 \%)$; (2) misunderstood importance $(28.3 \%)$; (3) oversight $(13.0 \%)$; (4) unavailability of test reservation in the nearest centers $(10.4 \%)$; (5) normal glycemic values at delivery $(8.3 \%)$; (6) discouragement by primary care physician (5.6\%). Conclusions: In our population, most women with recent GDM failed to perform ppOGTT. Our results indicated that the prominent barriers could potentially be overcome.
\end{abstract}

Keywords: gestational diabetes mellitus; ppOGTT; type 2 diabetes mellitus

\section{Introduction}

Type 2 diabetes mellitus (T2D) is the most common metabolic disease. It affects about $9 \%$ of the adult population [1], with an increasing global incidence due to the westernized lifestyle [2]. T2D is associated with long-term complications which greatly affect quality of life, reduce life expectancy, and contribute to the huge healthcare costs $[3,4]$. Therefore, it is mandatory to implement rigorous strategies to prevent T2D, to reduce its risks, and to address diabetic patients towards early and efficacious therapies.

Gestational diabetes mellitus (GDM), the type of glucose intolerance that develops during the second and third trimester of pregnancy [5], is a strong risk factor for the development of T2D later 
in life. In fact, T2D has a cumulative incidence of $60 \%$ at 10 years after a pregnancy, complicated by GDM [6]. Also, the worldwide incidence of GDM has greatly risen, affecting $20 \%$ of the pregnant women tested in some populations [7]. Many lines of evidence indicate that the identification of women with glucose intolerance during the postpartum period is of critical importance, as appropriate treatments can prevent or delay the onset of T2D [8-10]. Therefore, the most important medical societies all recommend screening for glucose intolerance in the early post-partum period in women with GDM [11]. Nevertheless, no consensus exists about when and how this screening should be performed [11]. In our country, current guidelines recommend that women with GDM have a 2-h $75 \mathrm{~g}$ oral glucose tolerance test (OGTT) 6 to 12 weeks after delivery (ppOGTT) [12]. However, despite compelling lines of evidence demonstrating the relevance of postpartum screening among women with previous GDM and the increased risk for non-adherent women in developing T2DM later in life, the rate of GDM women receiving appropriately-timed postnatal glucose testing is quite low [11]. Several explanations have emerged for the non-attendance of women for ppOGTT after GDM [13-18]. They include patient subjective barriers, such as newborn care, travel and socioeconomic difficulties, the lack of interest for healthcare, the lack of family support, the lack of understanding about the T2D risk and test discomfort [19]. They also include concerns regarding the healthcare system, including insufficient communication between gynecologists/diabetologists and primary care physicians, the perception among gynecologists, and primary care physicians that postpartum follow-up of GDM is not important, as well as the lack of universal guidelines [20]. In order to overcome barriers of testing, many interventions have been proposed in recent years [21]. Among them, it should be mentioned that verbal and written antepartum counselling [21,22], continuous postpartum follow-up [12], patient and physician reminders [14,23-27], flexible appointment times and dynamic roles in decision and planning of medical tests [28]. However, none of these strategies have obtained satisfying results [11]. We previously reported that adopting verbal and written antepartum counselling increased the follow-up screening rate from $24.1 \%$ to $62.4 \%$ in our population [22], where GDM prevalence is relatively high [29]. Unfortunately, this procedure, which has been derived from a specific healthcare project, has been stopped for economic reasons. Thus, we planned to retrospectively verify the adherence rate to ppOGTT and the reasons for non-adherence.

\section{Materials and Methods}

From January 2015 to December 2016, 1413 pregnant women underwent GDM screening at the Hospital "Pugliese-Ciaccio" in Catanzaro, Italy, in compliance with the Italian guidelines [30]. Out of the 1413 women, 451 (31.8\%) were diagnosed with GDM. The diagnosis of GDM was made in accordance with the IADPSG cut-off points (fasting $\geq 92 \mathrm{mg} / \mathrm{dl}, 1 \mathrm{~h} \geq 180 \mathrm{mg} / \mathrm{dl}, 2 \mathrm{~h} \geq 153 \mathrm{mg} / \mathrm{dl}$ ) [31]. Women with pre-existing type 1 or type 2 diabetes mellitus, as defined by ADA criteria [5], were excluded from the study. Anamnestic information included age, parity, family history of diabetes (first- or second-degree relatives), previous GDM, self-reported pre-pregnancy weight, pre-existing polycystic ovary syndrome (PCOS) (as defined by "The Rotterdam ESHRE/ASRM-sponsored PCOS consensus workshop group" criteria) [32], class of GDM risk (as defined by Italian guidelines) [30], insulin treatment. From all women, the information above were recorded at the time of GDM screening and during the gestation. This cohort has already been included in a previous work [33]. All interviews were conducted by the principal investigators by telephone and then transcribed. The interview was structured with closed-ended questions. For each case, only the main barrier was recorded. All women provided a verbal consent before the interview. The study was approved by the local ethics committee ( $\mathrm{n}^{\circ}$ 33, 27 June 2017).

After testing for normality of all continuous variables by the Shapiro-Wilk normality test, the non-parametric Mann-Whitney test was used for comparisons among the women performing or not-performing ppOGTT. The 2-tailed Fisher exact test was employed for comparison of proportions. Continuous variables are expressed as median and interquartile range (IQR), and categorical variables 
as numbers and percentages. In all analyses, statistical significance was fixed at an alpha level of 0.05 . All calculations were performed with SPSS 20.0 software (SPSS Inc, Chicago, IL, USA).

\section{Results}

We were able to contact 327 (72.4\%) out of 451 pregnant women with recent GDM. Among them, $230(73.3 \%)$ did not perform ppOGTT, and only 97 (29.7\%) were compliant with the Italian guidelines. As shown in Table 1, the return rate was higher in women classified as "high risk" for GDM during pregnancy, in those undergoing early GDM screening, with familial history of T2D in first grade relatives, with previous GDM, with middle/high educational status, and with PCOS. No difference was observed when age, pre-pregnancy BMI, gravidity, and insulin treatment were considered.

Table 1. Characteristics of the enrolled women who had, or had not performed ppOGTT.

\begin{tabular}{lccc}
\hline \multicolumn{1}{c}{ Maternal Characteristics } & ppOGTT & No ppOGTT & \multirow{2}{*}{$\boldsymbol{p}$ Value } \\
\cline { 2 - 3 } & $\boldsymbol{n}=\mathbf{9 7}$ & $\boldsymbol{n = 2 3 0}$ & 0.8842 \\
\hline Age, yr & $33(30-36)$ & $34(30-36)$ & 0.6229 \\
Pregravidic BMI, kg/m ${ }^{2}$ & $25.9(22.3-28.3)$ & $25.1(22.4-28.2)$ & 0.0101 \\
Familial history of type 2 diabetes, $n$ & $52(53.6 \%)$ & $87(37.8 \%)$ & 0.026 \\
Previous GDM, $n$ & $21(21.6 \%)$ & $27(11.7 \%)$ & 0.6464 \\
Gravidity, $n$ & $2(1-2)$ & $2(1-2)$ & $<0.0001$ \\
Middle/high educational status, $n$ & $71(73.2 \%)$ & $105(45.6 \%)$ & 0.0004 \\
PCOS, $n$ & $17(17.5 \%)$ & $11(4.8 \%)$ & $<0.0001$ \\
High risk women ${ }^{*}, n$ & $47(48.4 \%)$ & $42(18.3 \%)$ & $<0.0001$ \\
Early GDM screening ${ }^{*}, n$ & $58(59.8 \%)$ & $31(13.5 \%)$ & 0.0917 \\
Insulin treatment, $n$ & $38(39.2 \%)$ & $67(29.1 \%)$ & \\
\hline
\end{tabular}

Data are medians (interquartile range in brackets) for age, pre-gestational BMI, and gravidity or numbers $(n)$. $\mathrm{P}$ values refer to overall differences across groups as derived from non-parametric Mann Whitney test or Fisher's exact test, respectively. Middle/high educational status: Secondary school/university degree. * According to the Italian guidelines, high risk women are those with at least one of the following parameters: previous GDM, pre-pregnancy body mass index (BMI) $\geq 30 \mathrm{~kg} / \mathrm{m}^{2}$, fasting plasma glucose (FPG) at first visit or before pregnancy between $100-125 \mathrm{mg} / \mathrm{dL}$ (5.6-6.9 mmol/L). For these women, a 75-g $2 \mathrm{~h}$-OGTT is recommended early in pregnancy (16-18 weeks) [30].

During the phone interviews, the women that had not performed ppOGTT were asked for the reasons of their poor compliance, and all their answers were classified into 6 groups (Table 2). As reported in Table 2, the first barrier that prevented postnatal screening was newborn care. In fact, $30.4 \%$ of women claimed that the need for continuous baby care was the main reason that had prevented them from ppOGTT testing. The second barrier was due to their poor understanding of the importance of this test $(28.3 \%)$. Then, $30(13.0 \%)$ cases admitted that they had overseen it; 24 $(10.4 \%)$ women were unable to obtain a ppOGTT reservation in the nearest centers. In Italy, the public healthcare system requires a reservation to have access to any health service, but the waiting lists are often very long. Of the cohort, 19 (8.3\%) women believed that the normalization of glycemic values after the delivery made the ppOGTT unnecessary. Surprisingly, $13(5.6 \%)$ women claimed that they have been discouraged by their primary care physician. When we searched a correlation between the different barriers and the women's features, we observed that, among the above-mentioned barriers, newborn care was more frequent among women with a GDM requiring insulin treatment compared with insulin-untreated women $(31 / 67$ versus $39 / 163, p=0.0015)$. No other correlation was observed. 
Table 2. Prominent barriers to ppOGTT.

\begin{tabular}{ccc}
\hline Barrier Reference Number & Barrier & $\boldsymbol{n}=\mathbf{2 3 0}$ \\
\hline 1 & Newborn care & $70(30.4 \%)$ \\
2 & Misunderstood the importance of ppOGTT & $65(28.3 \%)$ \\
3 & Overseen & $30(13.0 \%)$ \\
4 & Unavailability of ppOGTT reservation in the nearest centers & $24(10.4 \%)$ \\
5 & Normal glycemic values after delivery & $19(8.3 \%)$ \\
6 & Advised against by primary care physician & $13(5.6 \%)$ \\
\hline
\end{tabular}

Data are numbers $(n)$.

\section{Discussion}

Current Italian guidelines recommend ppOGTT in women with GDM. However, we found that the adherence to this test has decreased since antepartum counseling has been left out [22]. Herein, we investigated the barriers to pPOGTT in Italian women, after the interruption of verbal antepartum counseling [22]. We first observed that 70.3\% (230/327) of women with recent GDM did not perform ppOGTT. That is, the rate of women performing ppOGTT has been halved with respect to that observed when counselling was introduced [22], thus confirming the critical importance of this tool in our population. The investigation of obstacles to ppOGTT indicated that a prominent barrier was represented by a competing priority, that is newborn care. This data is consistent with other reports and, interestingly, it is common to any examined population [18,34,35]. In addition, it is worth noting that the priority towards the newborn constitutes a prevailing barrier even in women treated with insulin during pregnancy, a category that generally demonstrates a stronger compliance for ppOGTT.

Similar to other reports, our findings indicated that the lack of patient understanding and awareness of the risks of T2DM and the low motivation for self-care are relevant reasons for non-adherence to ppOGTT [22,36-39]. In fact, in our study, over 50\% of answers (answers 2), 3), and 5)) can be attributed to these reasons. This is consistent with the observations that women with middle/high educational status and/or with a previous clinical condition are predisposed to T2DM susceptibility (e.g., previous GDM, PCOS, high risk for GDM), all factors already stimulating a greater awareness, were more compliant. Furthermore, this study is consistent with previous data, from us and others, indicating a marked improvement in return rate after appropriate counselling or other education tools [11]. The difficulty in obtaining a ppOGTT reservation in the nearest centers is a structural obstacle, very frequent in Southern Italy, that is related to the effectiveness and efficiency of healthcare system organization. However, we believe that even this barrier may be at least in part affected by the lack of awareness and low motivation for self-care. Inadequate communication between obstetrician and primary care physician, the perception that postpartum follow-up of GDM is not a clinical priority, and the lack of agreed protocols/procedures may be responsible for the last barrier $[39,40]$. Since failure to perform ppOGTT testing may potentially prevent the onset or delay of the diagnosis of T2DM in women who experienced GDM, and since compliance may be overcome by antepartum counselling, efforts should be made to encourage this practice in Southern Italy.

\section{Conclusions}

In our population, most women with recent GDM failed to perform the ppOGTT testing. The lack of patient understanding and awareness of the risks leading to T2DM, the low motivation for self-care and inadequate counselling by primary care physicians are contribute to non-adherence. All these barriers can be potentially overcome. Based on this study, we have promoted a patient's specific informative consultation close to term pregnancy and provided information protocols on pPOGTT to primary care physicians of our district. Further studies are needed to evaluate the efficacy of these methods for improving patients' compliance, and to establish whether counselling may be a good practice that could be generalized to other populations. 
Author Contributions: P.Q. and F.V. designed the study and recruited patients; E.C. contributed to the study design, analyzed data and wrote the manuscript; L.P. and R.V. (Roberta Venturella) recruited patients; D.P.F. and A.B. contributed to the critical revision of the article for important intellectual content; R.V. (Raffaella Vero) and C.D.C. supervised the study. All authors discussed the results and commented on the manuscript.

Funding: This research received no external funding.

Acknowledgments: We thank the staff of the Diabetes Unit of the Azienda Ospedaliera Pugliese-Ciaccio of Catanzaro, Italy, particularly M. Zangari, C. Lombardo, T. Marghella, V. Cristofaro, and G. Procopio, for their precious help in the recruitment and examination of patients.

Conflicts of Interest: The authors declare no conflict of interest.

\section{References}

1. Unwin, N.; Whiting, D.; Gan, D.; Jacqmain, O.; Ghyoot, G. IDF Diabetes Atlas—4th Edition: Epidemiology and Morbidity, Prevalence and Projections, 2010 and 2030. Available online: https:/ /www.idf.org/e-library/ epidemiology-research/diabetes-atlas/21-atlas-4th-edition.html (accessed on 13 November 2018).

2. Shaw, J.E.; Sicree, R.A.; Zimmet, P.Z. Global estimates of the prevalence of diabetes for 2010 and 2030. Diab. Res. Clin. Pract. 2010, 87, 4-14. [CrossRef] [PubMed]

3. Li, R.; Zhang, P.; Barker, L.E.; Chowdhury, F.M.; Zhang, X. Cost-effectiveness of interventions to prevent and control diabetes mellitus: A systematic review. Diabetes Care 2010, 33, 1872-1894. [CrossRef] [PubMed]

4. Zhang, P.; Zhang, X.; Brown, J.; Vistisen, D.; Sicree, R.; Shaw, J.; Nichols, G. Global healthcare expenditure on diabetes for 2010 and 2030. Diabetes Res. Clin. Pract. 2010, 87, 293-301. [CrossRef] [PubMed]

5. American Diabetes Association. Standards of medical care in diabetes-2015, 2. Classification and diagnosis of diabetes. Diabetes Care 2015, 38 (Suppl. 1), S8-S16.

6. Bellamy, L.; Casas, J.P.; Hingorani, A.D.; Williams, D. Type 2 diabetes mellitus after gestational diabetes: A systematic review and meta-analysis. Lancet 2009, 373, 1773-1779. [CrossRef]

7. Chiefari, E.; Arcidiacono, B.; Foti, D.; Brunetti, A. Gestational diabetes mellitus: An updated overview. J. Endocrinol. Investig. 2017, 40, 899-909. [CrossRef] [PubMed]

8. Retnakaran, R.; Qi, Y.; Sermer, M.; Connelly, P.W.; Zinman, B.; Hanley, A.J. Comparison of National Diabetes Data Group and American Diabetes Association diagnostic criteria for gestational diabetes in their identification of postpartum risk of glucose intolerance. Diabetes Res. Clin. Pract. 2009, 85, 40-46. [CrossRef]

9. Kim, C. Managing women with gestational diabetes mellitus in the postnatal period. Diabetes Obes. Metab. 2010, 12, 20-25. [CrossRef]

10. McClean, S.; Farrar, D.; Kelly, C.A.; Tuffnell, D.J.; Whitelaw, D.C. The importance of postpartum glucose tolerance testing after pregnancies complicated by gestational diabetes. Diabet. Med. 2010, 27, 650-654. [CrossRef]

11. Pastore, I.; Chiefari, E.; Vero, R.; Brunetti, A. Postpartum glucose intolerance: An updated overview. Endocrine 2018, 59, 481-494. [CrossRef]

12. Metzger, B.E.; Buchanan, T.A.; Coustan, D.R.; de Leiva, A.; Dunger, D.B.; Hadden, D.R.; Hod, M.; Kitzmiller, J.L.; Kjos, S.L.; Oats, J.N.; et al. Summary and recommendations of the Fifth International Workshop-Conference on Gestational Diabetes Mellitus. Diabetes Care 2007, 30 (Suppl. 2), S251-S260. [CrossRef]

13. Chamberlain, C.; McLean, A.; Oats, J.; Oldenburg, B.; Eades, S.; Sinha, A.; Wolfe, R. Low rates of postpartum glucose screening among indigenous and non-indigenous women in Australia with gestational diabetes. Matern. Child Health J. 2015, 19, 651-663. [CrossRef] [PubMed]

14. Korpi-Hyovalti, E.; Laaksonen, D.E.; Schwab, U.; Heinonen, S.; Niskanen, L. How can we increase postpartum glucose screening in women at high risk for gestational diabetes mellitus? Int. J. Endocrinol. 2012, 2012, 519267. [CrossRef] [PubMed]

15. Tang, J.W.; Foster, K.E.; Pumarino, J.; Ackermann, R.T.; Peaceman, A.M.; Cameron, K.A. Perspectives on prevention of type 2 diabetes after gestational diabetes: A qualitative study of Hispanic, African-American and White women. Matern. Child Health J. 2015, 19, 1526-1534. [CrossRef] [PubMed]

16. Baker, A.M.; Brody, S.C.; Salisbury, K.; Schectman, R.; Hartmann, K.E. Postpartum glucose tolerance screening in women with gestational diabetes in the state of North Carolina. N. C. Med. J. 2009, 70, 14-19. [PubMed] 
17. Gupta, Y.; Gupta, A. Post-partum screening after gestational diabetes. Lancet Diabetes Endocrinol. 2013, 1, 90-101. [CrossRef]

18. Aziz, S.; Munim, T.F.; Fatima, S.S. Post-partum follow-up of women with gestational diabetes mellitus: Effectiveness, determinants, and barriers. J. Matern. Fetal Neonatal Med. 2017, 5, 1-6. [CrossRef]

19. Nielsen, K.K.; Kapur, A.; Damm, P.; de Courten, M.; Bygbjerg, I.C. From screening to postpartum follow-up-The determinants and barriers for gestational diabetes mellitus (GDM) services, a systematic review. BMC Pregnancy Childbirth 2014, 14, 41. [CrossRef]

20. Pierce, M.; Modder, J.; Mortagy, I.; Springett, A.; Hughes, H.; Baldeweg, S. Missed opportunities for diabetes prevention: Post-pregnancy follow-up of women with gestational diabetes mellitus in England. $\mathrm{Br}$. J. Gen. Pract. 2011, 61, e611-e619. [CrossRef] [PubMed]

21. Stasenko, M.; Liddell, J.; Cheng, Y.W.; Sparks, T.N.; Killion, M.; Caughey, A.B. Patient counseling increases postpartum follow-up in women with gestational diabetes mellitus. Am. J. Obstet. Gynecol. 2011, 204, 522.e1-6. [CrossRef]

22. Capula, C.; Chiefari, E.; Vero, A.; Iiritano, S.; Arcidiacono, B.; Puccio, L.; Pullano, V.; Foti, D.; Brunetti, A.; Vero, R. Predictors of postpartum glucose tolerance testing in Italian women with gestational diabetes mellitus. ISRN Endocrinol. 2013, 2013, 182505. [CrossRef] [PubMed]

23. Lega, I.C.; McLaughlin, H.; Coroneos, M.; Handley-Derry, F.; Donovan, N.; Lipscombe, L.L. A physician reminder to improve postpartum diabetes screening in women with gestational diabetes mellitus. Diabetes Res. Clin. Pract. 2012, 95, 352-735. [CrossRef] [PubMed]

24. Halperin, I.J.; Sehgal, P.; Lowe, J.; Hladunewich, M.; Wong, B.M. Increasing Timely Postpartum Oral Glucose Tolerance Test Completion in Women with Gestational Diabetes: A Quality-Improvement Initiative. Can. J. Diabetes 2015, 39, 451-456. [CrossRef] [PubMed]

25. Clark, H.D.; Graham, I.D.; Karovitch, A.; Keely, E.J. Do postal reminders increase postpartum screening of diabetes mellitus in women with gestational diabetes mellitus? A randomized controlled trial. Am. J. Obstet. Gynecol. 2009, 200, 634.e1-7. [CrossRef] [PubMed]

26. Heatley, E.; Middleton, P.; Hague, W.; Crowther, C. The DIAMIND study: Postpartum SMS reminders to women who have had gestational diabetes mellitus to test for type 2 diabetes: A randomised controlled trial-Study protocol. BMC Pregnancy Childbirth 2013, 13, 92. [CrossRef] [PubMed]

27. Vesco, K.K.; Dietz, P.M.; Bulkley, J.; Bruce, F.C.; Callaghan, W.M.; England, L.; Kimes, T.; Bachman, D.J.; Hartinger, K.J.; Hornbrook, M.C. A system-based intervention to improve postpartum diabetes screening among women with gestational diabetes. Am. J. Obstet. Gynecol. 2012, 207, 283.e1-6. [CrossRef] [PubMed]

28. Suan, M.A.M. Return for Postpartum Oral Glucose Tolerance Test Following Gestational Diabetes Mellitus. Asia Pac. J. Public Health 2015, 27, 601-609. [CrossRef] [PubMed]

29. Capula, C.; Chiefari, E.; Borelli, M.; Oliverio, R.; Vero, A.; Foti, D.; Puccio, L.; Vero, R.; Brunetti, A. A new predictive tool for the early risk assessment of gestational diabetes mellitus. Prim. Care Diabetes 2016, 10, 315-323. [CrossRef] [PubMed]

30. Linea guida Gravidanza fisiologica. Sistema nazionale per le linee guida dell'Istituto Superiore di Sanità. Available online: http:/ / www.snlg-iss.it/cms/files/Lg.Gravidanza.pdf (accessed on 13 March 2018).

31. International Association of Diabetes and Pregnancy Study Groups Consensus Panel. International Association of Diabetes and Pregnancy Study Groups Recommendations on the Diagnosis and Classification of Hyperglycemia in Pregnancy. Diabetes Care 2010, 33, 676-682. [CrossRef] [PubMed]

32. Rotterdam ESHRE/ASRM-Sponsored PCOS Consensus Workshop Group. Revised 2003 consensus on diagnostic criteria and long-term health risks related to polycystic ovary syndrome. Fertil. Steril. 2004, 81, 19-25. [CrossRef]

33. Chiefari, E.; Pastore, I.; Puccio, L.; Caroleo, P.; Oliverio, R.; Vero, A.; Foti, D.P.; Vero, R.; Brunetti, A. Impact of seasonality in gestational diabetes mellitus. Endocr. Metab. Immune Disord. Drug Targets 2017, 17, $246-252$. [CrossRef] [PubMed]

34. Chang, Y.; Chen, X.; Cui, H.; Zhang, Z.; Cheng, L. Follow-up of postpartum women with gestational diabetes mellitus (GDM). Diabetes Res. Clin. Pract. 2014, 106, 236-240. [CrossRef]

35. Lie, M.L.; Hayes, L.; Lewis-Barned, N.J.; May, C.; White, M.; Bell, R. Preventing type 2 diabetes after gestational diabetes: Women's experiences and implications for diabetes prevention interventions. Diabetic Med. 2013, 30, 986-993. [CrossRef] [PubMed] 
36. Tovar, A.; Chasan-Taber, L.; Eggleston, E.; Oken, E. Postpartum screening for diabetes among women with a history of gestational diabetes mellitus. Prev. Chronic Dis. 2011, 8, A124. [PubMed]

37. Kwong, S.; Mitchell, R.S.; Senior, P.A.; Chik, C.L. Postpartum diabetes screening: Adherence rate and the performance of fasting plasma glucose versus oral glucose tolerance test. Diabetes Care 2009, 32, 2242-2244. [CrossRef] [PubMed]

38. Ferrara, A.; Peng, T.; Kim, C. Trends in postpartum diabetes screening and subsequent diabetes and impaired fasting glucose among women with histories of gestational diabetes mellitus: A report from the Translating Research into Action for Diabetes (TRIAD) Study. Diabetes Care 2009, 32, 269-274. [CrossRef] [PubMed]

39. Bernstein, J.A.; McCloskey, L.; Gebel, C.M.; Iverson, R.E.; Lee-Parritz, A. Lost opportunities to prevent early onset type 2 diabetes mellitus after a pregnancy complicated by gestational diabetes. BMJ Open Diabetes Res. Care 2016, 4, e000250. [CrossRef] [PubMed]

40. Capula, C.; Chiefari, E.; Vero, A.; Foti, D.P.; Brunetti, A.; Vero, R. Prevalence and predictors of postpartum glucose intolerance in Italian women with gestational diabetes mellitus. Diabetes Res. Clin. Pract. 2014, 105, 223-230. [CrossRef] [PubMed]

(C) 2018 by the authors. Licensee MDPI, Basel, Switzerland. This article is an open access article distributed under the terms and conditions of the Creative Commons Attribution (CC BY) license (http:/ / creativecommons.org/licenses/by/4.0/). 